\title{
Warm Spitzer IRAC photometry: dependencies on observing mode and exposure time
}

\author{
Jessica E. Krick $\odot,{ }^{\text {a, } *}$ Patrick J. Lowrance $\odot,{ }^{\text {a }}$ Sean Carey, ${ }^{\text {a }}$ \\ Jason Surace, ${ }^{\mathrm{a}}$ Carl J. Grillmair, ${ }^{\mathrm{a}}$ Seppo Laine $\odot,{ }^{\mathrm{a}}$ \\ Schuyler D. Van Dyk $\odot,{ }^{a}$ James G. Ingalls $\odot,{ }^{a}$ Matthew L. N. Ashby, \\ and S. P. Willner ${ }^{\mathrm{b}}$ \\ ${ }^{a}$ Caltech, IPAC, Pasadena, California, United States \\ ${ }^{\mathrm{b}}$ Center for Astrophysics I Harvard \& Smithsonian, Cambridge, \\ Massachusetts, United States
}

\begin{abstract}
We investigate differences in Spitzer/IRAC 3.6 and $4.5 \mu \mathrm{m}$ photometry that depend on observing strategy. Using archival calibration data, we perform an in-depth examination of the measured flux densities (fluxes) of 10 calibration stars, observed with all the possible observing strategies. We then quantify differences in the measured fluxes as a function of (1) array mode (full or subarray), (2) exposure time, and (3) dithering versus staring observations. We find that the median fluxes measured for sources observed using the full array are $1.6 \%$ and $1 \%$ lower than those observed with the subarray at [3.6] and [4.5], respectively. In addition, we found a dependence on the exposure time such that for [3.6] observations, the long frame times are measured to be lower than the short frame times by a median value of $3.4 \%$ in full array and $2.9 \%$ in subarray. For [4.5] observations, the longer frame times are $0.6 \%$ and $1.5 \%$ in full and subarray, respectively. These very small variations will likely only affect science users who require high-precision photometry from multiple different observing modes. We find no statistically significant difference for fluxes obtained with dithered and staring modes. When considering all stars in the sample, the fractional well depth of the pixel is correlated with the different observed fluxes. We speculate the cause to be a small nonlinearity in the pixels at the lowest well depths where deviations from linearity were previously assumed to be negligible. (C) The Authors. Published by SPIE under a Creative Commons Attribution 4.0 International License. Distribution or reproduction of this work in whole or in part requires full attribution of the original publication, including its DOI. [DOI: 10.1117/1.JATIS.7.3.038006]
\end{abstract}

Keywords: infrared; space telescopes; instrumentation; detectors; photometry.

Paper 21056 received May 7, 2021; accepted for publication Sep. 10, 2021; published online Sep. 27, 2021.

\section{Introduction}

The Infrared Array Camera (IRAC) ${ }^{1}$ was operational on the Spitzer Space Telescope $^{2}$ from 2003 to 2020 with four broad mid-infrared bands with response covering 3.15 to $9.25 \mu \mathrm{m}$ (at 3.6, 4.5, 5.8, and $8.0 \mu \mathrm{m}$; also denoted channels 1 to 4 , respectively). These filter names are labels and not the actual effective wavelengths (for more detailed information on filter transmission see Ref. 3). After depletion of the cryogens, from mid 2009 until January 2020, only [3.6] and [4.5] were available for observations.

To efficiently calibrate the telescope, a discrete set of observing templates was enabled for IRAC observers. Available options to define the templates included array mode, frame time, and dithering strategy. The array mode could be specified either as full array (meaning, the entire IRAC $256 \times 256$ array of 1.2 arcsecond pixels was read out after each exposure) or subarray, in which case 64 consecutive $32 \times 32$ pixel images were taken at a higher readout rate without moving the telescope (0.01-s single readout for subarray versus 0.2 -s for full array). A number of preset frame times were available; those and their corresponding exposure times are listed in

*Address all correspondence to Jessica E. Krick, jkrick@ caltech.edu 
Table 1 Available IRAC frame times.

\begin{tabular}{lcc}
\hline \hline Frame time (s) & Exposure time (s) & Observing mode \\
\hline 0.02 & 0.01 & Subarray \\
0.1 & 0.08 & Subarray \\
0.4 & 0.36 & Subarray \\
2 & 1.92 & Subarray \\
0.4 & 0.2 & Full array \\
2 & 1.2 & Full array \\
6 & 4.4 & Full array \\
12 & 10.4 & Full array \\
30 & $(23.6,26.8)^{\mathrm{a}}$ & Full array \\
100 & $(93.6,96.8)$ & Full array \\
\hline \hline
\end{tabular}

Table 1. Frame time is a measure of time elapsed between successive array resets. The exposure time (effective integration times) is the time elapsed between the first pedestal and the first signal read, not including resets or multiple reads. High dynamic-range exposures, in which paired short and long exposures were acquired sequentially within a single full-array observing template, were also available to IRAC observers, but did not add diversity to the range of possible observations. Lastly, observers were able to choose from a discrete set of dither patterns, make their own mapping strategy, or employ a staring mode. Dithering (small position changes to reduce noise) and mapping (position changes of order the size of the array to cover an area greater than the size of the array) were both possible in the full array mode. Dithering was available in the subarray mode between 64-frame sets, but not within the sets. Observations that do not move positions between frames are known as "staring-mode." Staring mode was most often used for high-precision time-series observations of brown dwarfs and exoplanets.

The rich Spitzer/IRAC archive contains many observations of the same target taken with different array modes, frame times, or dithering strategies. This is often the case not only for serendipitous observations but also for observations designed intentionally in different modes. Possible science cases for this include, but are not limited to, (1) initial dithered observations to find proper motions of brown dwarfs followed by staring-mode data to refine the characterization of their atmospheres, (2) dithered debris disk searches looking for IR excess followed by a staring-mode study of variations in specific debris disks, (3) archival dithered observations followed by targeted subarray staring observations for confirmation, or (4) observations that had either a too low signal-to-noise ratio (SNR) or were saturated at one exposure time were followed up later in the mission using a different exposure time. These science cases therefore require combining photometry from different modes to arrive at scientific conclusions.

In this work, we examine how IRAC photometry depends on observing strategy. We emphasize that these are very small measured variations (a few percent at most) and so will likely only affect science users who require high-precision photometry from multiple different observing modes. A full set of calibration observations to test for variations in measured fluxes among observing modes were only taken during the warm IRAC mission. We consequently do not discuss data taken during the cryogenic mission (which ended in 2009) or in the [5.8] or [8.0] channels.

In Sec. 2, we describe the archival data used for this project. Section 2.2 covers our methods for reducing the data, carrying out photometry, and applying photometric corrections. Section 3 discusses the different potential correlations and photometry effects. We make concluding remarks in Sec. 4. 


\section{Data}

\subsection{Observations}

In this work, we used the calibration observations taken for Spitzer Program ID (PID) 1336 and PID 1367. Specifically, we observed a set of 10 stars with varying exposure times, in both subarray and in full array, and in staring and dithering modes. Almost always, staring-mode observations were taken on the same pixel (the sweet spot), as that pixel was the best characterized pixel on the array. Table 2 lists the stars. We searched the archive for additional data usable for this analysis but did not find anything suitable, combining nonvariable stars with observations in all available modes and having a sufficient number of images to achieve statistical significance.

Figure 1 shows a visualization of this dataset. We plot the exposure time(s) versus aperture flux for just the [4.5] channel. Similar observations were made for [3.6]. All stars were observed with multiple exposure times. Not all of the 10 stars could be observed at all exposure times due to SNR and saturation concerns. This plot illustrates how the range of stellar brightnesses and frame times in the sample filled the available phase space.

We considered how close to saturation (well depth) a star is as a means of interpreting our results. Looking at our sample as a whole, the range of possible well depths is not well sampled; having a median fractional well depth of 0.04 and 0.02 at [3.6] and [4.5], where a fractional well depth of 1.0 indicates saturation. Figure 1(b) shows well depths of the sample. The median well depths of our sample correspond to an SNR of 39 and 24 at [3.6] and [4.5], respectively. This dataset was not designed with well depth in mind and was instead designed to find stars that would sample the available exposure time parameter space.

Although available, we reject the 0.02 -s photometry because of its large scatter. We have also rejected any data where the well depth is greater than the listed saturation limit in the IRAC Instrument Handbook. ${ }^{4}$ The saturation limit results in the rejection of around 250 photometry points at [4.5]. All observations used in this work have SNRs of six or greater.

For a subset of three stars, we made a larger number of observations in all possible modes with 2-s frame times in both channels. This allowed us to build up statistically meaningful samples where we hold the frame time constant while varying the other observing parameters. The three stars are NPM1+66.0584, NPM1+66.0578, and KF03T2.

The 2-s frame time is the closest set of IRAC frame times available for holding exposure time constant between the full and subarray. Unfortunately, the 2-s subarray and 2-s full-array frame times do not actually correspond to the same exposure times: 1.92 and $1.2 \mathrm{~s}$, respectively. Similarly, frame times of $0.4 \mathrm{~s}$ in full and subarray also have different exposure times, so frame

Table 2 The calibration star sample.

\begin{tabular}{|c|c|c|c|c|c|c|}
\hline Name & $\begin{array}{l}\text { R.A. } \\
\text { (h m s) }\end{array}$ & Dec. (deg' ") & $\begin{array}{l}\text { Spec. } \\
\text { type }\end{array}$ & $\begin{array}{l}{[3.6]} \\
\text { (mJy) }\end{array}$ & $\begin{array}{l}{[4.5]} \\
(\mathrm{mJy})\end{array}$ & $\begin{array}{c}\text { Alternate } \\
\text { name }\end{array}$ \\
\hline NPM1+61.0569 & 172325.9 & +611240.7 & K0.5 III & 363 & 208 & - \\
\hline NPM1+74.0514 & 190253.5 & +741443.6 & K0.5 III & 323 & 194 & BD+74 804 \\
\hline NPM1+61.0582 & 173655.6 & +614058.1 & K1 III & 274 & 162 & - \\
\hline NPM1+66.0507 & 173122.1 & +664635.3 & K2 III & 250 & 145 & - \\
\hline NPM1+66.0578 & 192532.2 & +66 4738 & K1 III & 127 & 75 & - \\
\hline NPM1+57.0835 & 180412.6 & +574218.6 & - & 95 & 54 & - \\
\hline TYC4213-241-1 & 180345.53 & +66 5603.7 & K1 III & 74 & 42 & KF01T3 \\
\hline KF03T2 & 175751.4 & +663103.0 & K1.5 III & 70 & 40 & - \\
\hline NPM1+66.0584 & 193607.39 & +66 2154.2 & FO & 56 & 35 & BD+66 1222 \\
\hline NPM1+60.0581 & 172452.3 & +6025 50.8 & $\mathrm{~A} 1 \mathrm{~V}$ & 37 & 23 & BD+60 1753 \\
\hline
\end{tabular}


(a)

(b)

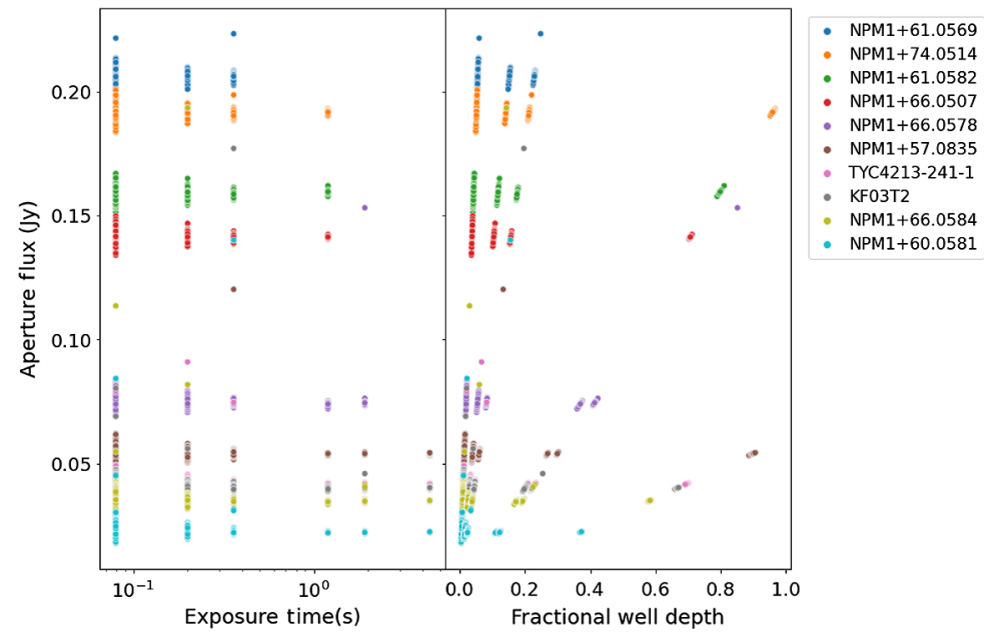

Fig. 1 Description of the dataset used in this work for the [4.5] channel, showing (a) the aperture flux as a function of the exposure time and (b) aperture flux as a function of well depth. The [3.6] channel has a similar observation set but for brevity is not shown. Points are color coded by star names, and symbol shapes denote full (filled circles) or subarray (crosses). Each data point represents one observation, and data are not binned, but there are overlapping data points.

time was not a good alternative. This difference in the exposure times for the same frame time comes from a different number of Fowler numbers $(N)$, wait ticks, and readout times for the different modes as listed in the IRAC Instrument Handbook. Briefly, Fowler sampling is a way of observing by taking $N$ nondestructive reads at the beginning of the observation and another $N$ nondestructive reads at the end of the observation, the difference of which is the flux measured per pixel.

All of the stars chosen for this work were vetted as potential calibration stars for IRAC. Seven of the 10 stars are published as primary or secondary calibrators for IRAC known to not exhibit flux variability in the IRAC bands. ${ }^{5}$ The three stars not included in that reference are NPM1 $+74.0514, \mathrm{NPM} 1+57.0835$, and NPM1+66.0584. Specifically, NPM1+66.0584 is one of the three stars in the subset taken with more observations in 2-s frame times. We cannot use this dataset to determine both if the stars are time variable, and if they vary as a function of the other parameters studied herein. While vetted, because these have not specifically been published as calibration stars, we experimented with removing these three stars from our sample. All plots look similar (albeit with larger scatter due to fewer data points), and conclusions remain the same if we remove those stars from the sample. We therefore choose to keep these in the sample for the remainder of this work.

Each of the [3.6] and [4.5] datasets includes about 80,000 total individual observations. Specifically, for each channel, we have roughly 65,000 subarray and 15,000 full array observations.

\subsection{Photometry}

We briefly describe our pipeline here, emphasizing where it differs from previous work. For an overview of the Spitzer/IRAC absolute photometric calibration, see Refs. 5 and 6. Specifics of the photometry pipeline used here are included in Ref. 7. We used the basic calibrated data (BCD) FITS files (suffix bcd.fits) from the Spitzer Heritage Archive (SHA). These BCD files have had a dark correction, flat-field correction, linearity correction, and conversion to flux units already applied.

One somewhat new aspect of this work is that we apply a different dark correction to staringmode data than we do to dithered observations. Staring-mode data are processed in the standard pipeline in the same manner as dithered data, including using a dark image that was made by dithering. The delay time between frames affects the bias level in the frame (including in the dark); this is known as the "first-frame effect." Therefore, dithered observations will have different bias levels and patterns than staring-mode observations since staring-mode observations have 
shorter delay times between consecutive frames, although that pattern is constant if the delay time is constant. This effect adds both noise and systematics to the photometry. For this reason, dithered darks are inappropriate for staring-mode science frames when precision photometry is required.

We used PID 1345 to make our own staring-mode dark suite for all subarray frame times. We began by removing the dithered dark correction from the PID 1345 data. Because the dark correction is not the last correction made to the $\mathrm{BCD}$ files, care was taken to first back out the other corrections, apply the staring-mode dark, then reapply the other pipeline corrections. We then created a median image for each exposure time $(0.02,0.1,0.4,2.0 \mathrm{~s})$ and used this median frame as the staring-mode dark. Applying this new staring-mode dark to the staring-mode data has a measurable impact on derived fluxes. We recommend that anyone doing precision photometry with staring-mode data uses a staring-mode dark instead of the pipeline-provided, dithered dark. While the IRAC pipeline will not include these starting-mode darks, code is available for users to change which darks are used in a BCD frame on the contributed-code section of the Spitzer IRAC website. ${ }^{8}$

To measure flux, we use our appropriately dark-corrected BCD exposures and make the following corrections in order. We first convert images into units of electrons to enable a statistical calculation of uncertainties. Second, we use a center-of-light method to find stellar centroids. ${ }^{9}$ Third, we do aperture photometry with a three-pixel radius aperture and (3-7) pixel background annulus. The small aperture size is chosen to reduce noise and the number of cosmic rays in the aperture. Fourth, we make a correction for pixel-phase using pixel_phase_correct gauss.pro. ${ }^{10}$ The pixel-phase correction accounts for gain changes as a function of the position within a pixel, coupled with the undersampling of a point source by IRAC. Fifth, we make a correction for array location. The array location-dependent correction takes into account the variation in system response of the instrument across the field of view, which is primarily due to the change in the angle of incidence of light through the bandpass filter as a function of position on the array. Lastly, we discard the first frame of every subarray FITS file and of every full array AOR. These frames are affected by the first-frame effect discussed above and are likely to have measured fluxes that differ from those of subsequent images. We do not apply an aperture correction since the same aperture is used for all photometry regardless of observing mode.

To compare photometry for all stars on the same plots, we normalize the stars to the same absolute level by dividing all photometry by the median stellar fluxes. The distributions of fluxes per star are somewhat skewed, so a mean does not capture the peak of the distribution. Having skewed distributions causes the mean levels in the subsequent plots to differ from unity.

\section{Results and Discussion}

This section describes how the measured IRAC photometry differs as a function of observing mode and exposure time. Section 3.3 examines the effects of observing mode and exposure time together. Finally, we consider the impact of staring and dithering on IRAC photometry.

We choose to present our results mainly with box plots to show the distributions per star as a function of both array mode (full or subarray) and exposure time for both channels, discussed individually in Secs. 3.1 and 3.2. A box plot shows the median of the distribution as the solid line in the middle of the box. The box top and bottom indicate the top and bottom quartiles, and the caps at the end of the lines show the maximum and minimum values in the distributions. These box plots include the entire dataset (all stars, all exposure times, full and subarray, staring and dithering). On average, each box contains a few thousand data points. In general, the boxes with smaller quartile ranges and $\mathrm{max} / \mathrm{min}$ values have fewer data points. The plots are color-coded by star for each of the 10 stars. Stars are listed in order from the brightest (NPM1+61.0569) to the faintest (NPM1+60.0581). Color-coding is consistent for all box plots and labeled in Fig. 2.

\subsection{Array Mode-Full versus Subarray}

Figure 2 shows the [3.6] and [4.5] photometry (top and bottom respectively) of the stars in Table 2 as a function of array mode, revealing that the measured fluxes appear on average 

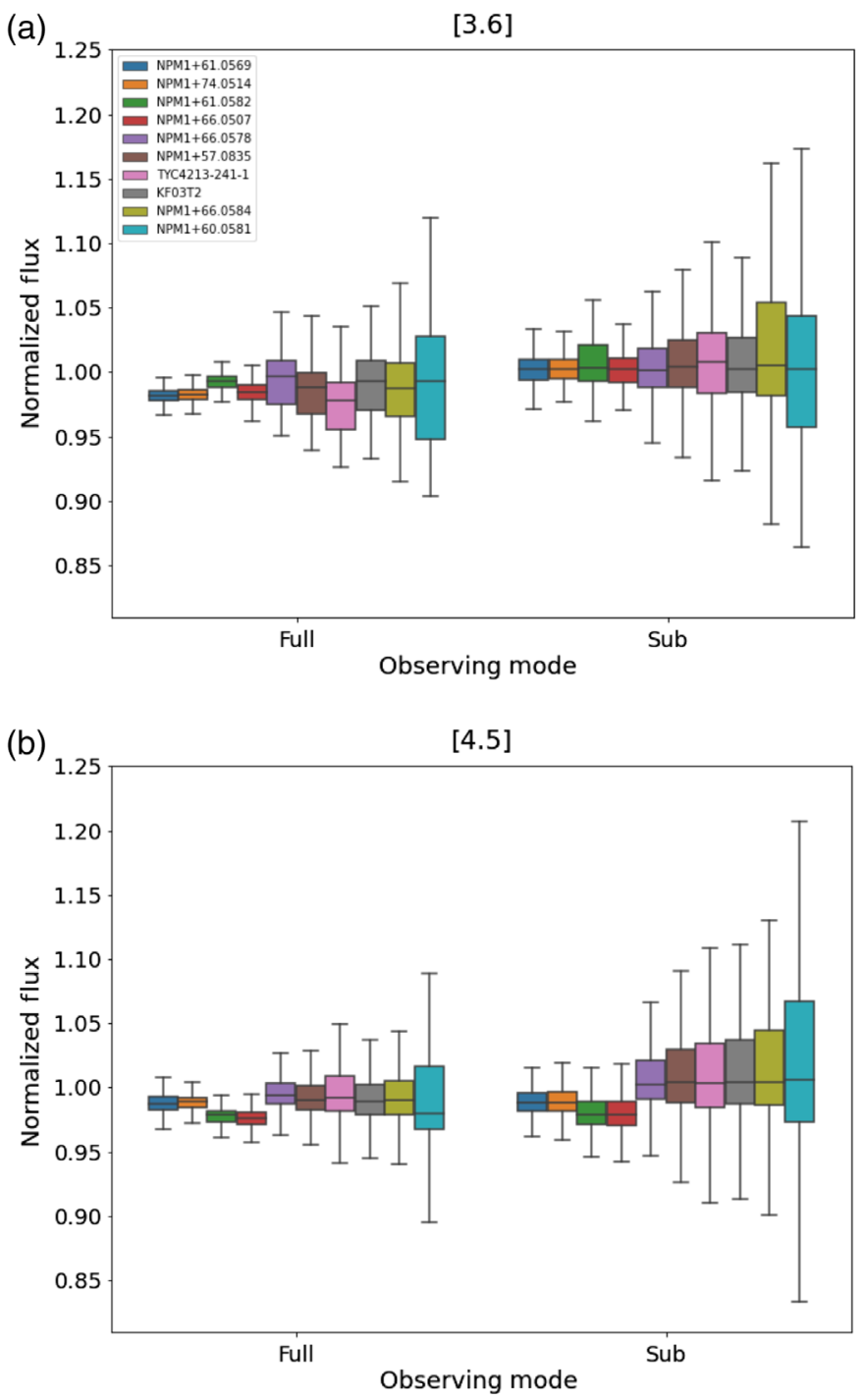

Fig. 2 Box plot of the normalized fluxes of all the stars divided into full and subarray observations for (a) [3.6] and (b) [4.5]. Stars are normalized by dividing by the median flux. The solid line in the middle of the boxes shows a median value, whereas the top and bottom of the box are quartiles, and the endcaps indicate the full range of the data. Stars are color coded according to the legend and listed in order of brightness from brightest to faintest. On average, the measured fluxes in fullarray observations are smaller than the measured fluxes in the subarray observations.

to be lower for the full-array observations than the subarray observations of the same stars. We attempt to confirm this statistically using an Anderson two-sample test, per star, to see if the distributions for full array and subarray are drawn from the same population. This statistical method considers the vertical distance between the two cumulative distributions. For all 10 stars, we can say that the full array and subarray data are not drawn from the same distribution at the $25 \%$ significance level (maximum possible significance). Thus, the difference between the fluxes measured in full array and subarray modes is statistically significant. Using all 10 stars, the median difference between the full and subarray flux measurements is $1.6 \%$ at [3.6] and $1.0 \%$ at $[4.5]$.

\subsection{Frame Time}

Figure 3 shows the distributions of normalized calibrator star fluxes as a function of frame time. [4.5] includes more data on the 6-s full-array frame time, so the bottom plot includes that frame 
(a) [3.6]

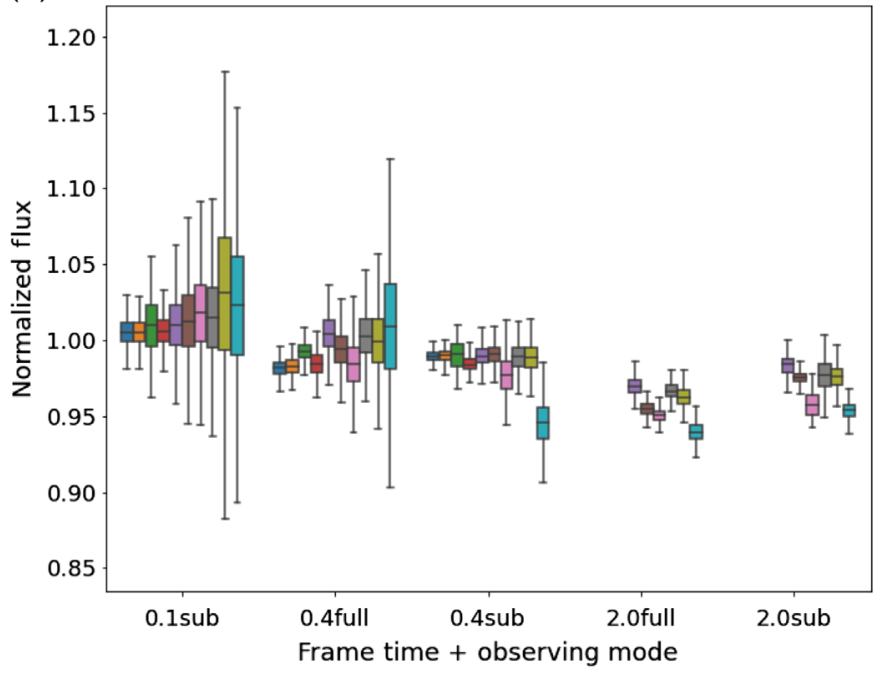

(b) [4.5]

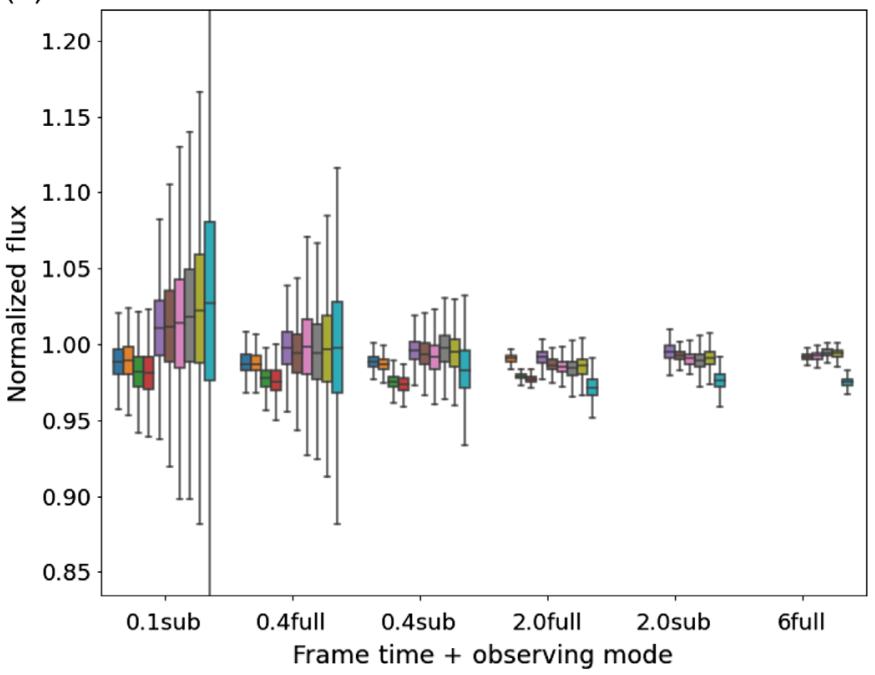

Fig. 3 Box plot of the distributions of normalized fluxes of all the stars as a function of exposure time at (a) [3.6] and (b) [4.5]. Frame time labels also indicate full or subarray. Color coding is the same as for Fig. 2. On average, fluxes measured at longer frame times are lower than those measured at shorter frame times.

time whereas the [3.6] plot does not. The median normalized flux of all the stars changes as a function of frame time such that the measured fluxes are larger at lower frame times. This is most strongly evident in [3.6]. Figure 4 shows a measure of the strength of this effect as the median difference between the 2-s and 0.4-s frame time fluxes for all the stars, per channel, per observing mode.

As in Sec. 3.1, we used the Anderson two-sample test to compare the full-array 0.4-s observations with the full-array 2-s observations. For both channels, we find the difference between the normalized flux distributions at the two frame times to be statistically significant. The same is true for fluxes from the subarray in 0.4- and 2-s frame time observations.

We tested whether the trend seen in flux as a function of exposure time depends on stellar brightness such that the brighter or the fainter stars would be more or less likely to show this effect. We do this by calculating the difference between the 2- and 0.4-s normalized fluxes. A difference between the fluxes implies that different fluxes are measured using different exposure times. Because we know there is an effect with array mode, we divide the sample into full and subarray data. Finally, looking for a trend in the stellar brightness, we divided the sample into 


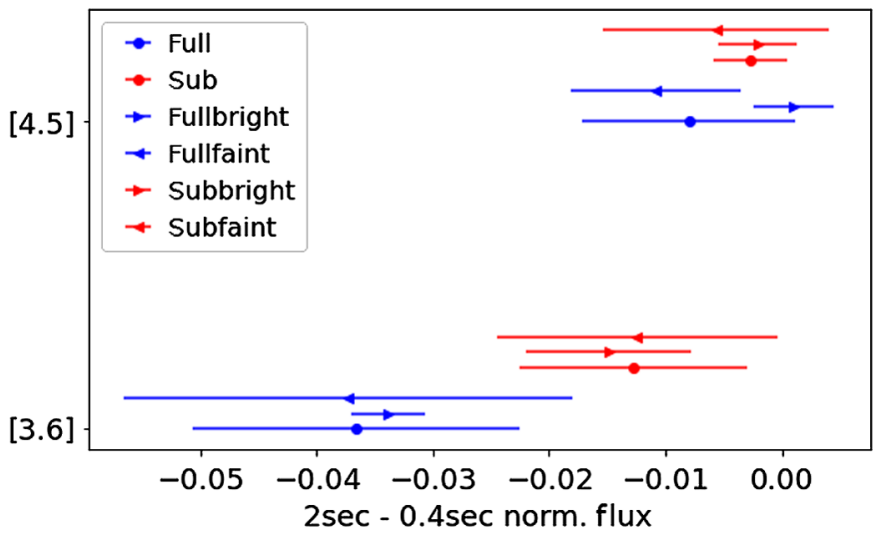

Fig. 4 Exposure-time effect for both channels divided into bright and faint stars to look for an effect with star brightness. [4.5] is shown in the top half of the diagram, while [3.6] plotted in the lower half. Blue circles show the median difference for all the stars observed in the full array between the 2- and 0.4-s frame time normalized fluxes, including one sigma error bars. Blue right and left triangles show the median values for the bright and faint populations of stars. The same quantities are plotted for subarray data in red.

bright and faint stars and recalculated the median values. Figure 4 shows the results, where the circular points are the median values of the difference in fluxes between the 2- and 0.4-s frametimes for both the full-array only data (blue) and the subarray only data (red). The bright and faint subsets are shown with the appropriately colored triangles. The differences between the bright and faint samples are within one sigma of each other. Here, sigma is calculated as the standard deviation between stars, which means that each bin contains only a few stars (and not thousands of data points). Our sample size is not large enough to make conclusions based on star brightness.

\subsection{Disentangling Exposure Time and Array Mode Effects}

We examine the possibility of disentangling the effects of exposure time and array mode. Instead of dividing the dataset by star, here we consider all stellar photometry as a single dataset and divide the dataset into four categories depending on the combination of array mode (full array versus subarray) and exposure time (short versus long). Each distribution has between 3000 and 50,000 stars (subarray exposure times have lots more frames than full array). Exact exposure times cannot be compared in this analysis because they differ significantly for full and subarray modes (see Table 1). For this reason, the division between the short and long exposure times is set at $0.3 \mathrm{~s}$ to construct statistically significant samples.

Figure 5 reveals differences between the overall distributions of photometry taken in full and subarray modes while accounting for exposure time, with enough data points to accurately reflect the shapes of the distributions. Longer exposure times are in red and light blue; shorter exposure times are in orange and dark blue. Especially at [3.6], an effect is apparent with both exposure time and observing mode. For the stars that have full-array observations in both short and long exposure times, the median difference in the flux is $3.4 \%$ at [3.6] (for six stars) and $0.6 \%$ at [4.5] (for 10 stars). For subarray, we find the median difference in flux between short and long exposure times to be $2.9 \%$ at [3.6] and $1.5 \%$ at [4.5].

\subsection{Well Depth}

The legend to Fig. 5 lists median well depths for each of the distributions. Well depth is one physical feature that correlates with the difference between short and long and full and subarray exposures. We do expect that longer exposure times on the same set of stars will have larger fractional well depths, so it makes sense that the full long and sublong distributions have the larger median well depths in both channels. Also, subarray mode has the possibility of shorter 
(a) [3.6]

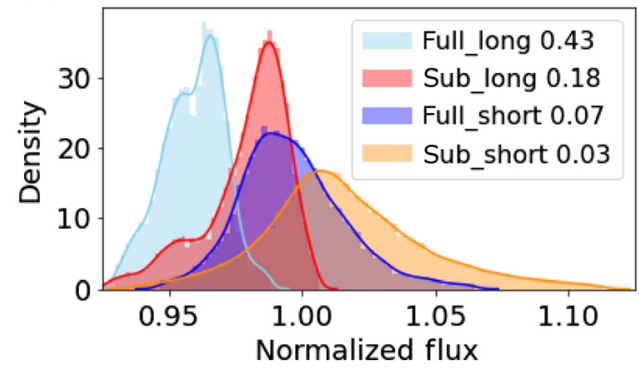

(b)

[4.5]

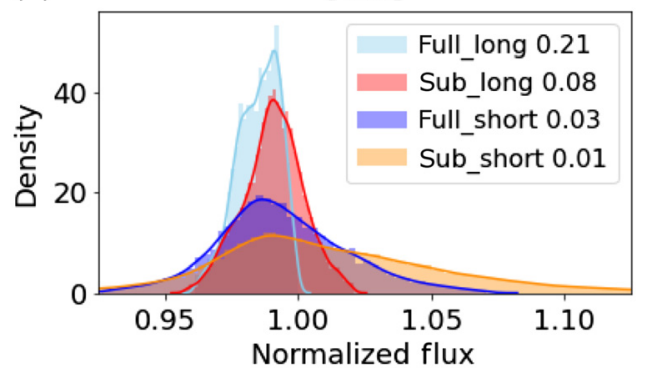

Fig. 5 Distribution of normalized star fluxes for (a) [3.6] and (b) [4.5]. The legend lists the different observing modes (full array versus subarray and long exposure times versus short exposure times) as well as the mean well depth per distribution. Solid dark lines show the kernel density estimation overplotted on the histograms. We see a difference in the absolute photometry of stars taken in available observing modes and exposure times shown here in the colored distributions.

(a) [3.6]

(b)

[4.5]

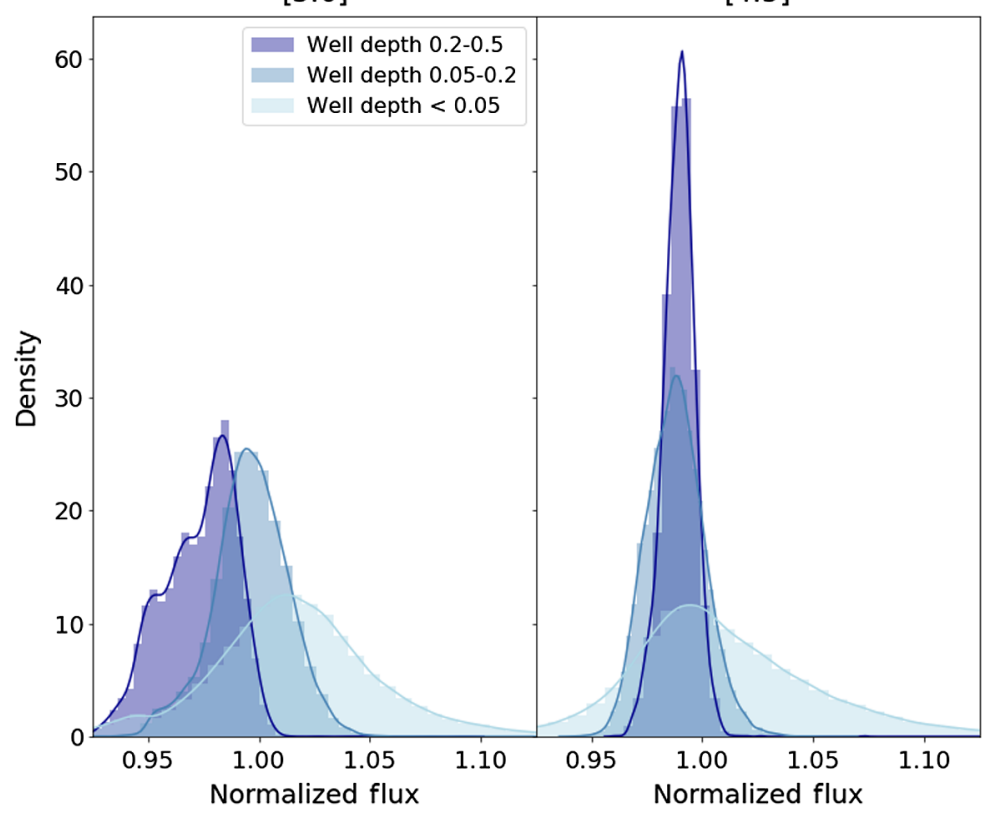

Fig. 6 Distributions of fluxes in three fractional well depth bins for (a) [3.6] and (b) [4.5]. Colors for the distributions get darker as the fractional well depth increases.

frame times than full array, so we would expect that subarray would have lower well depths than full array. Both channels show this behavior.

Figure 6 shows the distributions of normalized fluxes for the entire sample of stars in [3.6] and [4.5] divided into three well-depth bins. These bins do not have the same number of photometry points in them as we have many more low well-depth observations than intermediate 
well-depth observations. Because of this change in the number of data points per bin, we do not quote values for the peak of each bin, but rather address the trends revealed by the data in hand [3.6] shows a clear trend such that observations at intermediate well depths have lower normalized fluxes. [4.5] shows little, if any trend, consistent with trends seen in the observing mode and exposure time plots. While there are sources at higher well depth than $50 \%$ full well, their distribution overlaps those of the $20 \%$ to $50 \%$ bin. We conclude that only fractional well-depths less than a few tens of percent are affected by this well-depth effect. Consideration of well-depth does not help to disentangle the effects we have seen with observing mode and exposure time, but it is a clue that potentially the source of some of what we are seeing is nonlinearity in the low welldepth regime.

\subsection{Dithering versus Staring-Mode}

We do not detect a difference in photometry between the dithered and staring-mode observations. For three stars, we have observations in all the modes for the 2-s exposure times (substaring, subdithering, full staring, and full dithering). Recall that subarray observations taken with 2-s frame-time have 1.92-s exposure times and 2-s frame time full-array observations have 1.2-s effective exposure time, so the 2-s exposure time subarray and full array observations are not equivalent, but they are the closest to equivalent that exist in the archive.

Figure 7 shows a Cleveland dot plot of the median and standard deviation of the normalized fluxes for each mode, for each of the three stars in this sample. The legend also lists the number of data points per mode. The full and subarray measurements differ as before (see Sec. 3.1). The staring-mode photometry is consistent with the dithered mode photometry within one sigma in both channels.

\subsection{Multiple Regression}

Finding a relation among the observing parameters (array mode, exposure time, staring/dithering) may help both to (a) understand the effects found in this paper and (b) correct for them. We therefore use the statistical technique of multiple regression to search for any relations. Specifically, we used multiple linear regression, i.e., multiple independent variables, to predict the value of the dependent variable. To correct IRAC photometry for these effects, we have tried ordinary least squares (OLS) as a multiple linear-regression technique. We used array mode, exposure time, and stare/dither as independent variables, and flux as the dependent variable. We used two different modules in Python for this work: statsmodels ${ }^{11}$ and sklearn. ${ }^{12}$ We are unable to find successful models. The $R^{2}$ goodness of fit is 0.037 , when "good" models should have values close to 1.0. The failure of OLS in this situation could imply that the relationship between the independent and dependent variables is nonlinear.

(a)

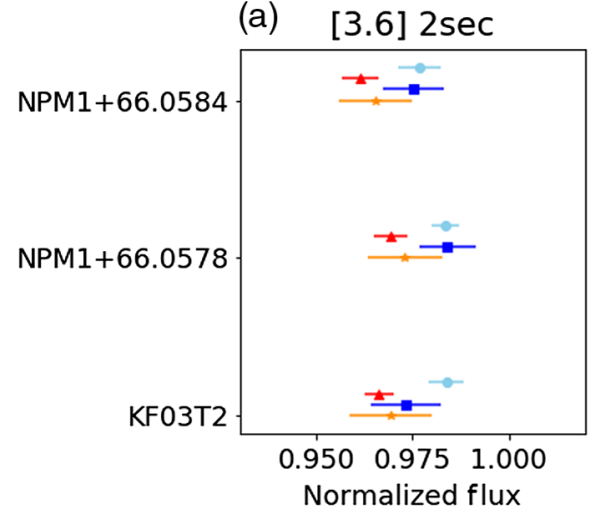

(b) $[4.5] 2 \mathrm{sec}$

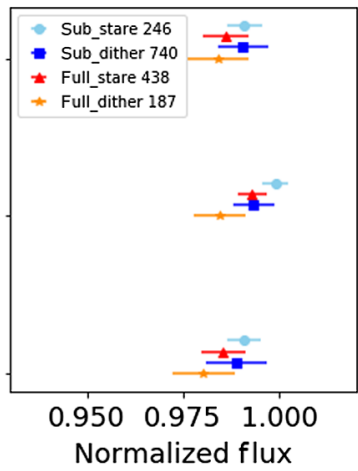

Fig. 7 Cleveland dot plot comparing normalized fluxes from different observing modes and dithering versus staring for three stars listed on the y-axis. (a) [3.6] and (b) [4.5]. The legend is for both plots and lists the number of data points for each mode. 


\section{Conclusions}

We document exposure time, array mode (full versus subarray), and fractional well depth flux dependence in IRAC photometry. The full array has median fluxes higher than the subarray by $1.6 \%$ at [3.6] and $1.0 \%$ at [4.5]. Dividing the sample further, the long exposures have a lower median flux than shorter exposures. The difference at full array is 3.4\% at [3.6] and $0.6 \%$ at [4.5]. For subarray, we find the median difference in flux is $2.9 \%$ at [3.6] and $1.5 \%$ at [4.5]. These noted effects are only relevant for a small fraction of IRAC high precision users with data in multiple modes who should include these values in their uncertainty calculations. We posit two potential causes of the noted low-level differences in photometry.

Overall, the normalized fluxes decrease as well depth increases up to a few tens of percent full well. Well depth is correlated with the differences in the flux distributions between full and subarray and long and short exposures. While these correlations with array mode and exposure time are expected, it potentially indicates the presence of nonlinearities at the low well depths sampled in this work.

Linearity corrections were made for warm IRAC to correct for a known effect where an increase in incoming photons does not correspond to an increase in counts (or data numbers DN). This occurs because filling the well decreases the potential, which in turn results in a less responsive system. The linearity correction for the IRAC InSb arrays inherently assumes no linearity correction at low well depths. Therefore, time-intensive observations were not made during the mission to include extremely low well-depth observations. Instead, calibration observations focused on the moderate ( $>20 \%$ ) to high well depths where the linearity deviated most significantly from a straight line correlation between photons and DN (see IRAC data handbook for a description of the derivation of the warm linearity correction). ${ }^{4}$ One explanation for why this low count linearity effect could exist at [3.6], but not [4.5] is that the applied biases are different between [3.6] and [4.5] implying that the electric potentials are different, which could explain the stronger effect at [3.6] than [4.5].

Understanding the root cause of nonlinearities is beyond the scope of this paper. Many complicating issues are hiding under that designation including the 3D structure of a pixel, how the linearity interacts with Fowler sampling (specifically how to translate corrections derived at one set of Fowler sampling parameters to those in another, which would get worse as the integration times become comparable to the time spent reading the detector), the speed at which the electric fields are changing in these very short exposures compared with the timescales of the exposures or the readouts and persistent image trap filling.

Linearity can in some detectors depend on the flux of the source in the sense that it matters not only how many photons come in to the detector but also the rate at which they fill the well. We do not have enough observations to know if the effect we are seeing is dependent on the brightness of the stars.

A second possible explanation for the difference between full and subarray photometry is that the array is resetting faster between consecutive frames for the subarray. A faster reset applies a stronger reverse bias on the array more frequently, which could affect the distribution of photoelectron traps, and therefore could have a low-level effect on photometry. We know that other similar effects (e.g., first-frame effect) are more significant at [3.6] than [4.5]. ${ }^{13}$

No flux difference is apparent between the staring and dithering mode observations after correcting the staring-mode data by a staring-mode dark. Anyone doing absolute photometry with staring-mode data should be using a staring-mode dark. The full and subarray staring-mode datapoints considered here are all taken on the same array pixel (the sweet spot of the subarray), which is not true for the dithered positions. Thus, residual pixel-phase uncertainties cannot be the cause of the measured flux differences between the full and subarray staring modes, otherwise we would see this effect in this work when comparing staring and dithering modes.

We have no evidence for low-level, persistent images being the cause of the differences in photometry measured here. We know that the persistent images are stronger at [3.6]. However, for [3.6] the same fraction of observations were taken at the sweet-spot pixel in both full and subarray images. The sweet spot is the pixel at the center of the array where most observations are conducted in the subarray, because it has been the best characterized for the pixel phase effect. This means that the sweet-spot pixel is more likely to have frequent low-level, persistent 
images than other pixels on the array. If a larger fraction of observations in the subarray had been taken at the sweet spot than the full array, we might have expected persistent images to be the culprit. On the contrary, we see no evidence for this.

Our analysis implies that these differences in fluxes are systematic; they do not average out with more observations. All full-array photometry will be different than all subarray photometry, no matter how many observations are taken in any given observing mode.

\section{Acknowledgments}

We thank the anonymous referees for their time and care in providing very useful comments on this manuscript. This work was based in part on observations made with the Spitzer Space Telescope, which is operated by the Jet Propulsion Laboratory, California Institute of Technology under a contract with NASA. This research has made use of NASA's Astrophysics Data System, the NASA/IPAC Infrared Science Archive, which is operated by the Jet Propulsion Laboratory, California Institute of Technology, under contract with the National Aeronautics and Space Administration, and the SIMBAD database, operated at CDS, Strasbourg, France. The acknowledgments were compiled using the Astronomy Acknowledgment Generator.

\section{References}

1. G. G. Fazio et al., "The Infrared Array Camera (IRAC) for the Spitzer space telescope," Astrophys. J. Suppl. 154, 10-17 (2004).

2. M. W. Werner et al., "The Spitzer space telescope mission," Astrophys. J. Suppl. 154, 1-9 (2004).

3. J. L. Hora et al., "Photometry using the infrared array camera on the Spitzer space telescope," Publ. Astron. Soc. Pac. 120, 1233 (2008).

4. S. Laine, "IRAC instrument handbook," https://irsa.ipac.caltech.edu/data/SPITZER/docs/ irac/iracinstrumenthandbook/ (2021).

5. W. T. Reach et al., "Absolute calibration of the infrared array camera on the Spitzer space telescope," Publ. Astron. Soc. Pac. 117, 978-990 (2005).

6. S. Carey et al., "Absolute photometric calibration of IRAC: lessons learned using nine years of flight data," Proc. SPIE 8442, $84421 \mathrm{Z}$ (2012).

7. J. E. Krick et al., "Spitzer IRAC photometry of JWST Calibration Stars," Astron. J. 161, 177 (2021).

8. J. E. Krick, "Change_dark_calibrate.pro," https://irsa.ipac.caltech.edu/data/SPITZER/docs/ dataanalysistools/tools/contributed/irac/change_dark_calibrate/ (2020).

9. S. J. Carey, J. G. Ingalls, and J. E. Krick, "Box_centroider.pro," https://irsa.ipac.caltech.edu/ data/SPITZER/docs/irac/calibrationfiles/pixelphase/box_centroider.pro (2020).

10. "Pixel_phase_correct_gauss.pro," https://irsa.ipac.caltech.edu/data/SPITZER/docs/irac/ warmfeatures/pixel_phase_correct_gauss.pro.

11. S. Seabold and J. Perktold, "Statsmodels: econometric and statistical modeling with Python," in 9th Python in Sci. Conf. (2010).

12. F. Pedregosa et al., "Scikit-learn: machine learning in Python," J. Mach. Learn. Res. 12, 2825-2830 (2011).

13. "Philosophy and Scope of the Spitzer Space Telescope Observer's Manual," https://irsa.ipac .caltech.edu/data/SPITZER/docs/files/spitzer/som12.2.pdf (2018).

Jessica E. Krick is an astronomer and data scientist on the Infrared Science Archive science platform team at Caltech/IPAC. She received her $\mathrm{PhD}$ in astronomy and astrophysics from the University of Michigan and has worked as part of the Spitzer IRAC instrument support team. Her scientific interests range from zodiacal light to exoplanets to clusters of galaxies and include machine learning and data science techniques applied to big astronomical datasets.

Patrick J. Lowrance is currently part of the ground system team of the Roman Space Telescope Science Support Center at IPAC. He received his PhD in astronomy and astrophysics from 
UCLA in 2001. He has worked with different aspects of the science and operations of spacebased observatories and instruments, including the Hubble and Spitzer Space Telescopes, for two decades.

Sean Carey is a senior staff scientist at Caltech/IPAC. He received his $\mathrm{PhD}$ in physics from RPI in 1995. He has worked on the data processing and calibration of the MSX Celestial Backgrounds data and the IRAC instrument aboard the Spitzer Space Telescope. He currently is manager of the NASA Exoplanet Science Institute at Caltech/IPAC and calibration scientist/ task lead for the NEO Surveyor Science Data System.

Jason Surace is an astronomer specializing in large areal surveys and observations of extreme star formation and active galactic nuclei, using advanced imaging techniques across a broad range of wavelengths but with a special emphasis on the infrared. He worked on the first adaptive optics systems on Mauna Kea, was the IPAC data system lead for PTF and ZTF, and in space worked on IRAS, WIRE, Spitzer, and currently the NEO Surveyor mission.

Carl J. Grillmair is a staff scientist at Caltech/IPAC. He received his $\mathrm{PhD}$ in astrophysics from Australian National University in 1993. He has worked on calibration and data processing for the Hubble Space Telescope, the Spitzer Space Telescope, and the WISE/NEOWISE missions.

Seppo Laine received his $\mathrm{PhD}$ from the University of Florida with a thesis on a barred galaxy NGC 7479. He held postdoctoral positions at the University of Hertfordshire, University of Kentucky, and at the Space Telescope Science Institute. Since 2002, he has been working at Caltech/IPAC, serving the Spitzer, Roman, and James Webb Space Telescopes, and assisting the IPAC Communications and Education Team. His scientific interests are in nearby galaxy structure, dynamics, and interactions.

Schuyler D. Van Dyk: Biography is not available.

James G. Ingalls is an associate staff scientist at IPAC at Caltech. Over nearly two decades, his primary duties centered on maintaining and improving the performance of astronomy instruments on board the Spitzer Space Telescope. He is currently leading the coronagraph instrument operations system on the Roman Space Telescope.

Matthew L. N. Ashby is an astrophysicist at the CfAlHarvard and Smithsonian, where he is a co-investigator on NASA's SPHEREx mission and codirector of NSF's Research Experience for Undergraduates at $\mathrm{SAO}$. He received his $\mathrm{PhD}$ in astronomy from Cornell University. He is a former member of Spitzer's IRAC instrument team, for which he served as mission operations and data analysis lead for more than a decade. His scientific interests lie in wide-field infrared surveys.

S. P. Willner is a research astronomer at the Center for Astrophysics. 